\title{
Radiofrequency ablation in the treatment of atypical cartilaginous tumours in the long bones: lessons learned from our experience
}

\author{
Edwin F. Dierselhuis ${ }^{1} \cdot$ Jelle Overbosch ${ }^{2} \cdot$ Thomas C. Kwee $^{2}$ - Albert J. H. Suurmeijer ${ }^{3}$. Joris J. W. Ploegmakers ${ }^{4}$. \\ Martin Stevens ${ }^{4} \cdot$ Paul C. Jutte ${ }^{4}$
}

Received: 16 June 2018 / Revised: 1 September 2018 / Accepted: 12 September 2018 / Published online: 29 September 2018

(C) The Author(s) 2018

\begin{abstract}
Background Surgery is the cornerstone of treatment of symptomatic cartilaginous neoplasms. We previously studied the application of radiofrequency ablation of atypical cartilaginous tumours in the long bones. The purpose of the present study was to investigate the additional effect of placing multiple needles and a longer procedure duration on the proportion of completely ablated tumours. Post-ablation MRI findings and the occurrence of complications were also assessed.

Methods We prospectively included 24 patients with atypical cartilaginous tumours in the long bones. Patients underwent CTguided radiofrequency ablation followed by curettage with adjuvant phenolisation 3 months later, retrieving material assessed for viable tumour. Before curettage, gadolinium-enhanced MRI was performed to check for residual tumour. The occurrence of complications was noted.

Results Complete tumour ablation was achieved in 17 out of 24 patients (71\%). Complete ablation was achieved in 5 of the 6 cases $(83 \%)$ when multiple needles were used in tumours $\geq 30 \mathrm{~mm}$. There was incomplete ablation in $8 \%$ of patients. Postablation gadolinium-enhanced MRI findings agreed with the histological results in 17 out of 23 cases and there was a negative predictive value of $83 \%$. One patient suffered a fracture after radiofrequency ablation.

Conclusion Radiofrequency ablation could be an alternative to curettage when treating atypical cartilaginous tumours in the long bones. It was shown that multiple needle placement in addition to longer duration of the ablation procedure is an effective measure in achieving complete ablation in tumours $\geq 30 \mathrm{~mm}$. Gadolinium-enhanced MRI has a negative predictive value of $83 \%$ and could guide post-ablation follow-up.
\end{abstract}

Keywords Atypical cartilaginous tumours $\cdot$ Low-grade chondrosarcoma $\cdot$ Radiofrequency ablation $\cdot$ Minimally invasive

\section{Introduction}

Atypical cartilaginous tumours (ACTs), also known as chondrosarcoma grade I, are bone tumours of borderline or

Edwin F. Dierselhuis

edwin.dierselhuis@radboudumc.nl

1 Department of Orthopaedics, Radboudumc, Postbus 9101, 6500 HB Nijmegen, the Netherlands

2 Department of Radiology, University of Groningen, University Medical Center Groningen, Groningen, The Netherlands

3 Department of Pathology and Medical Biology, University of Groningen, University Medical Center Groningen,

Groningen, The Netherlands

4 Department of Orthopaedics, University of Groningen, University Medical Center Groningen, Groningen, The Netherlands low malignant potential [1]. These lesions increasingly present as a coincidental finding, when patients are evaluated for other bone- or joint-related conditions [2-4]. ACTs are a type of cartilage-forming neoplasm, but unlike higher-grade tumours they do not generally metastasise $(<2 \%)$ and show excellent survival rates, with $<3 \%$ local recurrences [5]. Correct diagnosis in the past has been deemed rather difficult, as histological or radiological features alone are not always conclusive [6]. Consequently, tumour upgrading was seen in some cases of local recurrence. For this reason, wide resection of the bone and surrounding tissue used to be recommended sometimes even as primary treatment to avoid this risk [7]. However, recent literature shows that atypical cartilaginous tumours in the appendicular skeleton can be safely treated by curettage with adjuvant phenolisation or cryotherapy, provided that local recurrence rates are low $(0-7.7 \%)$ and have no negative effect on patient survival [5, 6, 8-16]. Application of 
this surgical technique has led to an improvement in functional results, although complications such as fracturing may still occur in up to $13 \%$ of cases $[6,8-13]$. In this context, the tumour biology should be weighed against the morbidity of intralesional surgery. For this reason, some favour a conservative approach, but data are scarce and only retrospective [17]. Minimally invasive treatment may be an alternative, with the advantage of local control, but largely without the burdens of conventional surgery.

In orthopaedic oncology there is increasing interest in the thermal ablation of bone tumours. Radiofrequency ablation (RFA) is a minimally invasive and highly accurate treatment tool. Thermal ablation by RFA has become the gold standard for treatment of certain benign bone tumours (i.e. osteoid osteomas) and can be advantageous in the treatment of skeletal metastases or solid organ tumours (i.e., renal cell carcinoma and hepatocellular carcinoma) [18-21].

In a previous proof-of-principle study by our group, ablation efficacy of RFA in ACTs was assessed by MRI and subsequent histological examination of ablated tumour tissue. Occurrence of complications and short-term functional outcome were also assessed [22]. Complete necrosis was achieved in $45 \%$ of patients, whereby size and localisation of the tumour were the main predictors of failure. This result was promising but not satisfactory. Tumours more than $30 \mathrm{~mm}$ in diameter were prone to incomplete ablation. The significance of the "heat sink" effect could not be demonstrated. We therefore altered the protocol so that tumours $\geq 30 \mathrm{~mm}$ were to be ablated using multiple needle placement. We also increased the amount of energy delivered with more ablation cycles. The purpose of the current study is to report on the effect of these measures on the proportion of completely ablated tumours, the correlation with post-ablation MRI findings and the occurrence of complications.

\section{Materials and methods}

\section{Design}

A prospective cohort study was conducted among patients with ACT in the long bones. Inclusion criteria were: patients aged $\geq 18$ with a diagnosis of ACT in the long bones on MRI (e.g. septonodular gadolinium enhancement, no or limited endosteal scalloping, no perilesional oedema), who opted for surgical intervention (Fig. 1). Other indications for surgery were growth of the tumour over time and/or persistent pain at the tumour site. Tumour size was limited to $50 \mathrm{~mm}$ maximum diameter in any plane. Tumours were not included if located in the hand, foot, pelvis or axial skeleton. Other exclusion criteria were the presence of cognitive impairments, cortical breakthrough and previous treatment of the same lesion. Written informed consent was obtained from all participants. The study was approved by the medical ethical review committee of our hospital (METc no. M09.077334). All procedures performed in studies involving human participants complied with the ethical standards of the institutional and/or national research committee and with the 1964 Declaration of Helsinki and its subsequent amendments or comparable ethical standards.

\section{Methods: ablation and surgical technique}

A CT-guided biopsy under general or spinal anaesthesia, followed by RFA in the same session, was conducted as previously reported [22]. Three months later, gadoliniumenhanced magnetic resonance imaging (Gd-MRI) was performed to assess for completeness of tumour ablation, followed within 4 weeks by curettage and adjuvant phenolisation. The ablation session was performed by one of our consultant musculoskeletal interventional radiologists using a Soloist ${ }^{\mathrm{TM}}$ Single Needle Electrode (Boston Scientific, Natick, MA, USA; Fig. 1). The session started with $2 \mathrm{~W}$, adding $1 \mathrm{~W}$ every minute, and ended automatically when the needle reached its point of roll-off due to highly elevated impedance of the ablated tissue. Multiple needle placement was applied when incomplete ablation was anticipated in tumours measuring $\geq 30 \mathrm{~mm}$ (Fig. 2). Material obtained during biopsy was examined by a pathologist with special expertise in bone and softtissue tumours (A.S). Patients were discharged from the hospital on the same day.

Curettage was performed in accordance with our usual procedure: a cortical window was created and the lesion was curetted (Fig. 3). After removal of the tumour, phenolisation of the cavity was carried out for $2 \mathrm{~min}$, followed by ethanol washout and saline rinsing. Polymethylmethacrylate (PMMA) was used to fill the defect in all cases. The retrieved material was sent to pathology for histological confirmation of ACT and assessment of the proportion of necrotic tumour tissue. All surgical procedures were performed by one of two orthopaedic oncologists (P.J. and J.P.).

\section{Pathology}

The endpoint was the success rate expressed as a percentage of patients in whom tumour ablation was complete on histology. Reaching $100 \%$ cell necrosis was regarded as a pR0 response. Subtotal $(95-99 \%)$ or incomplete $(<95 \%)$ tumour eradication were considered $\mathrm{pR} 1$ and $\mathrm{pR} 2$ respectively. Correlation with post-ablation Gd-MRI findings was noted, in addition to the occurrence of complications.

\section{Radiology}

Measurements of tumour size (largest diameter in any plane) were based on 4-mm slice MR images and 1.5-mm slice CTs. 
Fig. 1 a Representative MRI of an atypical cartilaginous tumour (ACT) in the proximal femur. b Transverse and $\mathbf{c}$ coronal images of CT-guided radiofrequency ablation (RFA) of the same tumour
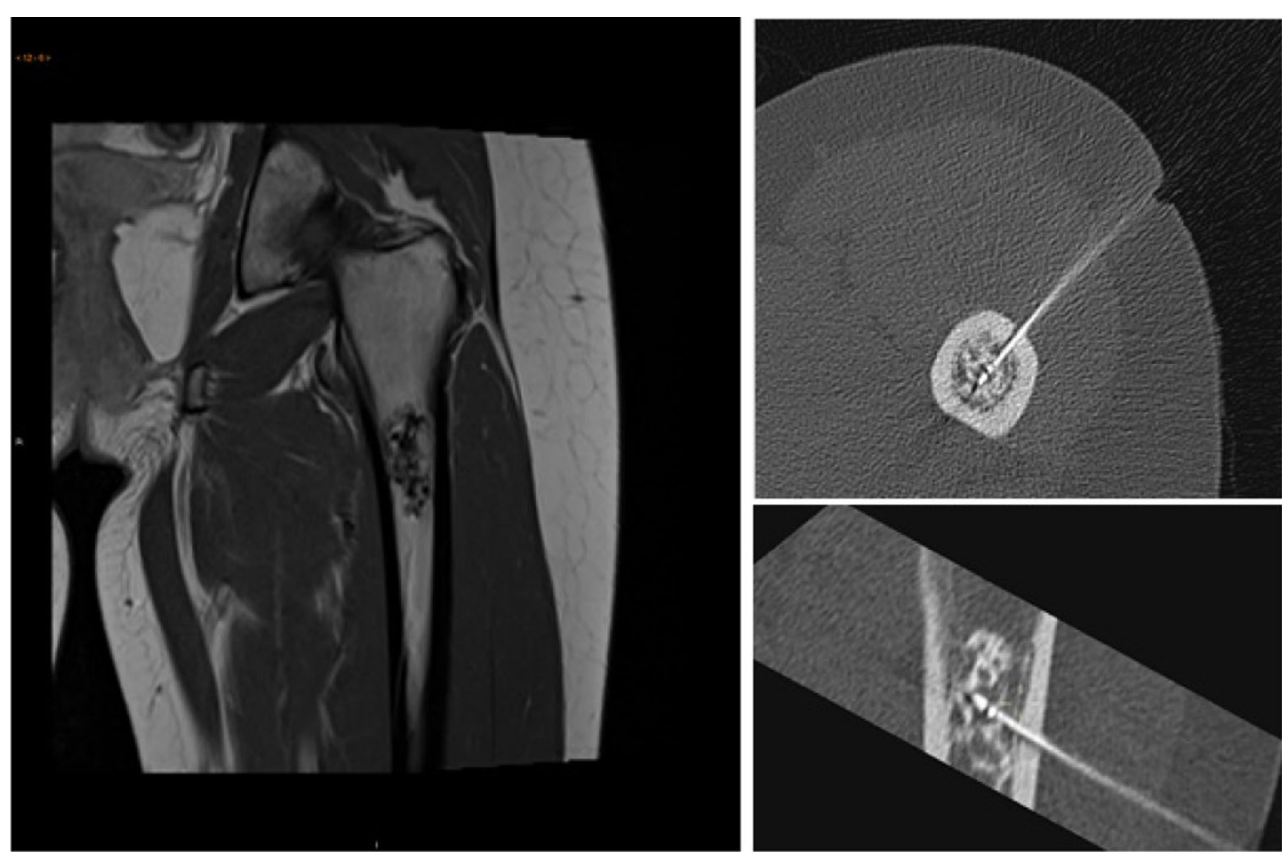

Imaging was also analysed using a grading system that included three categories: no signs of residual tumour (rR0), little or doubtful gadolinium uptake at the tumour border (rR1), and clear residual tumour outside the ablation zone (rR2). Needle positioning was assessed retrospectively. All post-RFA GdMRI were graded by a musculoskeletal radiologist (J.O.), who was blinded to the histological results.

\section{Statistical analysis}

Mean and range of values were noted for all variables. SPSS version 22.0 software (IBM-SPSS, Armonk, NY, USA) was used for all statistical testing. If applicable, a univariate analysis was undertaken using Student's $t$ test for normally distributed values and the Mann-Whitney $U$ test for non-parametric

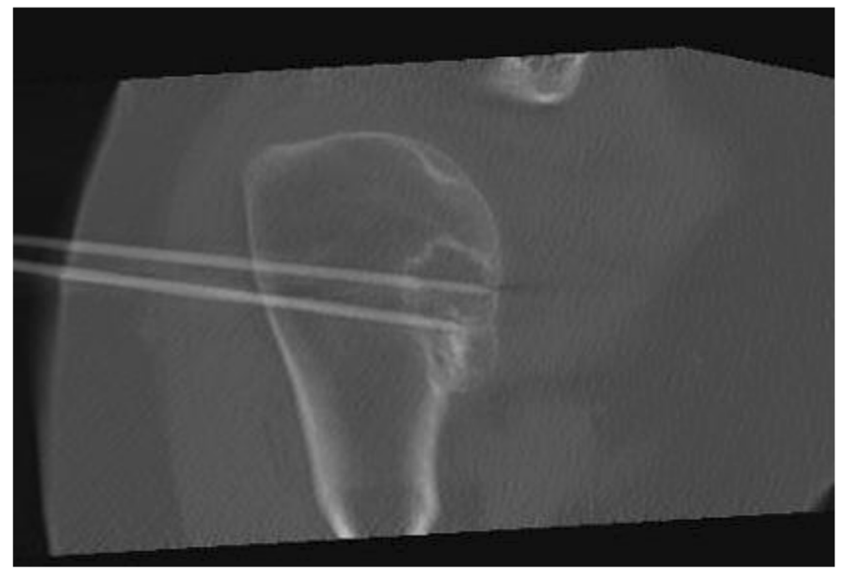

Fig. 2 Radiofrequency ablation procedure of an ACT in the proximal humerus with two-needle placement data; a $p$ value $<0.05$ was considered to be statistically significant.

\section{Results}

\section{Demographics}

In total, 24 patients were included, with a mean age of 51.1 years (range 31-75). The femur was affected most $(n=$ $16)$, followed by the humerus $(n=5)$ and tibia $(n=3)$. Mean tumour size was $28.3 \mathrm{~mm}$ (range 15-43). Six patients received multiple needle placement, all in tumours measuring $\geq 29 \mathrm{~mm}$. The RFA procedure took on average $23.6 \mathrm{~min}$ (range 12-37;

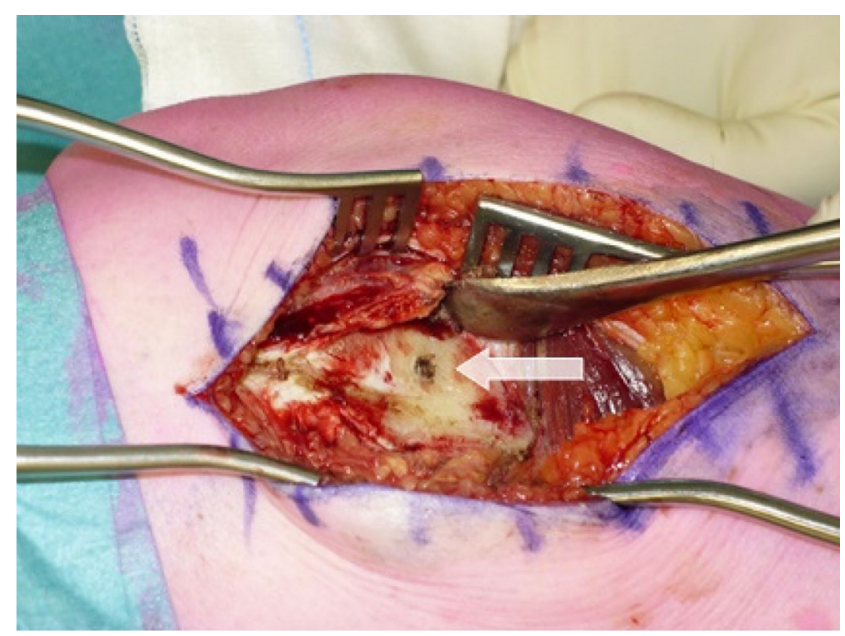

Fig. 3 Exposure of the bone to reach the tumour through a cortical window that has to be created. Note the scar from the previous insertion of an RFA needle (white arrow) 
Table 1 Patient characteristics and outcome

\begin{tabular}{|c|c|c|c|c|c|c|c|c|c|}
\hline $\begin{array}{l}\text { Case } \\
\text { number }\end{array}$ & Age (years) & Sex & Location & $\begin{array}{l}\text { Diameter } \\
(\mathrm{mm})\end{array}$ & $\begin{array}{l}\text { Ablation duration } \\
\text { (min) }\end{array}$ & Needles & Histological response & Radiological response & Complications \\
\hline 1 & 56 & Female & Femur (M) & 24 & 22 & Single & Focal residue & Focal uptake & None \\
\hline 2 & 63 & Female & Femur $(\mathrm{M})$ & 21 & 19 & Single & Complete necrosis & No uptake & None \\
\hline 3 & 51 & Female & Femur (M) & 30 & 25 & Single & Complete necrosis & No uptake & None \\
\hline 4 & 67 & Female & Femur (M) & 27 & 29 & Single & Complete necrosis & No uptake & None \\
\hline 5 & 49 & Female & Femur (D) & 35 & 23 & Multiple & Complete necrosis & No uptake & None \\
\hline 6 & 43 & Female & Humerus (D) & 15 & 19 & Single & Complete necrosis & No uptake & None \\
\hline 7 & 31 & Male & Femur (M) & 28 & 34 & Single & Focal residue & No uptake & None \\
\hline 8 & 52 & Female & Humerus (D) & 29 & 14 & Multiple & Complete necrosis & No uptake & None \\
\hline 9 & 46 & Female & Femur (M) & 31 & 21 & Single & Substantial residue & Substantial uptake & None \\
\hline 10 & 49 & Female & Humerus (D) & 29 & 29 & Single & Complete necrosis & Focal uptake & None \\
\hline 11 & 53 & Female & Tibia (D) & 24 & 26 & Single & Complete necrosis & No uptake & None \\
\hline 12 & 48 & Female & Femur (D) & 22 & 17 & Single & Complete necrosis & No uptake & None \\
\hline 13 & 63 & Female & Femur (D) & 29 & 37 & Single & Complete necrosis & - & Fracture \\
\hline 14 & 48 & Male & Femur (D) & 21 & 15 & Single & Complete necrosis & No uptake & None \\
\hline 15 & 58 & Female & Femur (M) & 24 & 12 & Single & Substantial residue & Focal uptake & None \\
\hline 16 & 63 & Male & Humerus (D) & 34 & 21 & Multiple & Complete necrosis & No uptake & None \\
\hline 17 & 40 & Female & Humerus (D) & 36 & 19 & Multiple & Complete necrosis & No uptake & None \\
\hline 18 & 59 & Female & Femur (D) & 36 & 14 & Multiple & Focal residue & No uptake & None \\
\hline 19 & 33 & Female & Femur (D) & 31 & 31 & Single & Complete necrosis & No uptake & None \\
\hline 20 & 31 & Male & Femur (D) & 30 & 21 & Single & Focal residue & Focal uptake & None \\
\hline 21 & 50 & Female & Tibia (D) & 43 & 31 & Multiple & Complete necrosis & No uptake & None \\
\hline 22 & 75 & Female & Femur (M) & 36 & 35 & Single & Complete necrosis & No uptake & None \\
\hline 23 & 49 & Female & Femur (M) & 26 & 27 & Single & Focal residue & No uptake & None \\
\hline 24 & 47 & Female & Tibia (D) & 19 & 25 & Single & Complete necrosis & No uptake & None \\
\hline
\end{tabular}

$M$ metaphysis, $D$ diaphysis

Table 1). In one patient, a Gd-MRI after RFA was not made, as a fracture occurred before the planned date of the scan. It was a low-energy fracture, 7 weeks after the index ablation procedure. Curettage was performed, followed by mini-open plate fixation without reduction. A non-union developed that needed second surgery with reduction, bone graft and plate fixation. The fracture healed well. This patient was still included for histological analysis purposes.

\section{Proportion of completely ablated tumours}

On a histological level, total ablation (pR0) was reached in 17 out of 24 cases $(71 \%)$. Incomplete ablation (pR2) was present in 2 out of $24(8 \%)$ and subtotal ablation (pR1) in 5 out 24 ( $21 \%)$ cases. In diaphyseal tumours, pR0 response was achieved in 13 out of $15(87 \%)$ cases compared with 4 out of 9 for metaphyseal tumours $(p=0.027)$. Duration of the ablation procedure was $24.4 \mathrm{~min}$ (range 14-37) in $\mathrm{pR} 0$, $23.6 \mathrm{~min}$ (range 14-34) in $\mathrm{pR} 1$ and $16.5 \mathrm{~min}$ (range 12-21) in $\mathrm{pR} 2$ cases $(p=\mathrm{NS})$.

\section{Correspondence with Gd-MRI}

Complete ablation was correctly diagnosed as rR 0 in 15 out of 16 cases, with the other case judged as rR1; pR1 corresponded with rR 1 in 2 out of 5 and $r R 0$ in 3 out of 5 cases respectively. The cases with a pR2 response were considered rR1 (1 out of 2) and rR2 (1 out of 2; Table 2).

\section{Needle positioning}

For tumours measuring $\leq 30 \mathrm{~mm}$ needle positioning was centric in 11 out of 14 cases, eccentric in 2 cases, and in 1 case multiple needles were applied in a $29-\mathrm{mm}$ diameter tumour. For tumours measuring $>30 \mathrm{~mm}$, needle placement was centric in 4 out of 10 cases, multiple needles were applied in 5 out of 10 cases, and in 1 case of a 31-mm tumour the needle was placed eccentrically (Fig. 4). In 1 out of 2 pR2 cases the needle was placed eccentrically (non-significant compared with pR0 and/or $\mathrm{pR} 1$ cases). In tumours measuring $>30 \mathrm{~mm}$, centric or multiple needle positioning led to $\mathrm{pR} 0$ in 7 out of 10 patients and pR1 in 2 out of 10 . This group had one pR2 in which the 
Table 2 Correlation of Gd-MRI with histological findings

\begin{tabular}{llllll}
\hline & & No uptake & $\begin{array}{l}\text { Gd-MRI } \\
\text { Focal uptake }\end{array}$ & Substantial uptake & Total \\
\hline \multirow{2}{*}{ pathology } & Complete necrosis & 15 & 1 & 0 & 16 \\
& Focal residue & 3 & 2 & 0 & 5 \\
& Substantial residue & 0 & 1 & 1 & 2 \\
\multirow{2}{*}{ Total } & 18 & 4 & 1 & 23 \\
\hline
\end{tabular}

needle was placed eccentrically. When multiple needles were used, complete ablation was achieved in all but 1 case.

\section{Discussion}

We demonstrated in $71 \%$ of patients that complete tumour necrosis is achievable using RFA for ACT in the long bones. Implementation of multiple needle placement in larger tumours and longer procedure duration improved ablation effectiveness. After our previous proof-of-principle study, we presented three possible causes of a failed ablation procedure:

1. Number and total ablation time of cycles

2. Tumour size $(>30 \mathrm{~mm})$

3. Heat sink effect

We slightly adjusted our study ablation technique by delivering more local energy, either by multiple needles or longer ablation duration [22]. We found that in all but 1 case complete ablation was achieved when multiple needles were used. Time is an issue, as temperature rise is a result of conductivity; hence, the longer the procedure takes, the more tissue is heated. Whether the heat sink effect plays a major role in the difference between success rates in metaphyseal and diaphyseal tumours is questionable, but more heat loss to surrounding tissue is plausible in metaphyseal bone if the thinness of

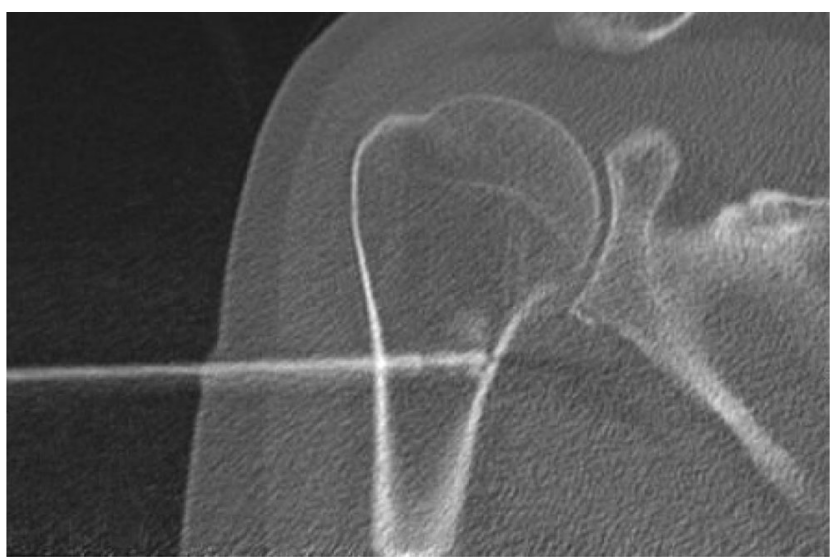

Fig. 4 Radiofrequency ablation procedure of ACT in the proximal humerus, with eccentric placement of the needle the cortex and the higher vascularity of the metaphysis are considered [23].

Although a quantitative comparison with our previous study was not performed, the achieved level of complete necrosis in $71 \%$ of the participants was higher than in the proofof-principle study (45\%), with a decrease in evident failures from 30 to $8 \%$ in the current study. Based on univariate analysis, diaphyseal tumours are most amenable for RFA treatment, with an $87 \%$ success rate. There were 2 patients with substantial viable tissue after ablation. In 1 case, the total ablation time was relatively short (12 $\mathrm{min}$ ). In the other case, the needle was placed eccentrically and, considering the size $(31 \mathrm{~mm})$, there should have been multiple needle placement. Both cases can thus be regarded as technical failures and were not conducted in accordance with our treatment protocol.

Post-ablation Gd-MRI findings corresponded with histological results in 17 out of 23 cases, with 5 cases understaged (the radiological response was better than the histology) and 1 case over-staged (the radiological response was worse than the histology). Fifteen out of 18 cases were correctly diagnosed as R0 on Gd-MRI (NPV $=83 \%)$. We want to stress that both failures (pR2) were seen on Gd-MRI, with one regarded as rR1. There is a chance of a small amount of residual tumour (pR1) being overlooked, but development of local recurrence (out of residue) is very gradual and has no negative effects on patient survival according to the current literature [5]. A recent paper has proposed a classification of MRI response after curettage with a consequent follow-up regime, which in our opinion can be extrapolated to MRI after ablation [24].

Despite the increased efficacy rates compared with our initial proof-of-principle study, there is still room for improvement. We are currently studying needle placement planning, in which ideally an algorithm can be developed using computer modelling and planning with computer-assisted surgery (CAS) to determine and execute optimal needle positioning, especially when multiple probe positions are used. Moreover, a needle that is regulated by temperature sensors instead of impedance could generate a more predictable ablation zone. Real-time imaging of the lesion during ablation would be of great value to monitor the ablation effect, albeit technically demanding. Needles used for thermal ablation are not MRIcompatible, and currently $\mathrm{CT}$ cannot detect temperature changes during RFA. Finally, an alternative might be the use 
of microwave ablation (MWA), as it is less dependent on tissue conductivity than RFA [25].

Our study also has some limitations. Only relatively small lesions were ablated and long-term follow-up after RFA is lacking. Some lesions might arguably have been enchondroma; yet, imaging and biopsy results convinced us of ACT in all cases. In addition, this study was designed as proof-of-principle for whether thermal ablation can treat chondroid tumours and to investigate the reliability of Gd-MRI to check for viable tumour post-ablation. For that reason, curettage served as a control for the effects of RFA at a histological level and assess correspondence of post-RFA histology with Gd-MRI. In the future, RFA will be investigated as a treatment tool instead of curettage, to draw definitive conclusions after adequate follow-up.

To summarise, we have demonstrated that RFA is capable of ablating ACTs in the long bones in $71 \%$ of cases, especially diaphyseal tumours. However, long-term follow-up is lacking and future studies should de designed to assess long-term outcome after RFA without subsequent curettage. It should be noted that for many years there has been a dearth of surgical innovations in the treatment of bone tumours, and we believe that the use of local tumour ablation can be a very valuable adjunct to current treatment options. We stress that not all ACTs are candidates for surgery, and yet there is neither a clear consensus on a conservative approach nor clear definitions of indications for surgery [17]. With this study, we have shown that multiple needle placement in addition to longer duration of the ablation procedure is an effective measure for achieving complete tumour ablation in tumours measuring $\geq 30 \mathrm{~mm}$. Gadolinium-enhanced MRI has a negative predictive value of $83 \%$ and could safely guide follow-up after RFA. Future studies should focus on planning, monitoring and further improving ablation efficacy by RFA technique, with adequate follow-up after the ablation procedure.

Acknowledgements We wish to thank Ruth Rose for her language assistance.

\section{Compliance with ethical standards}

Conflicts of interest The authors declare that they have no conflicts of interest.

Open Access This article is distributed under the terms of the Creative Commons Attribution 4.0 International License (http:// creativecommons.org/licenses/by/4.0/), which permits unrestricted use, distribution, and reproduction in any medium, provided you give appropriate credit to the original author(s) and the source, provide a link to the Creative Commons license, and indicate if changes were made.

\section{References}

1. Hogendoorn PB, Bovee JM, Nielsen GP. Chondrosarcoma (grades IIII), including primary and secondary variants and periosteal chondrosarcoma. In: Fletcher CDM, Bridge JA, Hogendoorn PCW,
Mertens F, editors. World Health Organization classification of tumours of soft tissue and bone, Vol. 5. Lyon: IARC; 2013. p. 264.

2. Hong ED, Carrino JA, Weber KL, Fayad LM. Prevalence of shoulder enchondromas on routine MR imaging. Clin Imaging. 2011;35(5):378-84.

3. Stomp W, Reijnierse M, Kloppenburg M, NEO study group, et al. Prevalence of cartilaginous tumours as an incidental finding on MRI of the knee. Eur Radiol. 2015;25(12):3480-7.

4. Kransdorf MJ, Peterson JJ, Bancroft LW. MR imaging of the knee: incidental osseous lesions. Radiol Clin N Am. 2007;45(6):943-5.

5. Hickey M, Farrokhyar F, Deheshi B, Turcotte R, Ghert M. A systematic review and meta-analysis of intralesional versus wide resection for intramedullary grade I chondrosarcoma of the extremities. Ann Surg Oncol. 2011;18(6):1705-9.

6. Leerapun T, Hugate RR, Inwards CY, Scully SP, Sim FH. Surgical management of conventional grade I chondrosarcoma of long bones. Clin Orthop Relat Res. 2007;463:166-72.

7. Eriksson AI, Schiller A, Mankin HJ. The management of chondrosarcoma of bone. Clin Orthop Relat Res. 1980;153:44-66.

8. Aarons C, Potter BK, Adams SC, Pitcher JD Jr, Temple HT. Extended intralesional treatment versus resection of low-grade chondrosarcomas. Clin Orthop Relat Res. 2009;467(8):2105-11.

9. Di Giorgio L, Touloupakis G, Vitullo F, Sodano L, Mastantuono M, Villani C. Intralesional curettage, with phenol and cement as adjuvants, for low-grade intramedullary chondrosarcoma of the long bones. Acta Orthop Belg. 2011;77(5):666-9.

10. Dierselhuis EF, Gerbers JG, Ploegmakers JJ, Stevens M, Suurmeijer AJ, Jutte PC. Local treatment with adjuvant therapy for central atypical cartilaginous tumors in the long bones: analysis of outcome and complications in one hundred and eight patients with a minimum follow-up of two years. J Bone Joint Surg Am. 2016;98(4):303-13.

11. Donati D, Colangeli S, Colangeli M, Di Bella C, Bertoni F. Surgical treatment of grade I central chondrosarcoma. Clin Orthop Relat Res. 2010;468(2):581-9.

12. Hanna SA, Whittingham-Jones P, Sewell MD, et al. Outcome of intralesional curettage for low-grade chondrosarcoma of long bones. Eur J Surg Oncol. 2009;35(12):1343-7.

13. Kim W, Han I, Kim EJ, Kang S, Kim HS. Outcomes of curettage and anhydrous alcohol adjuvant for low-grade chondrosarcoma of long bone. Surg Oncol. 2015;24(2):89-94.

14. Meftah M, Schult P, Henshaw RM. Long-term results of intralesional curettage and cryosurgery for treatment of low-grade chondrosarcoma. J Bone Joint Surg Am. 2013;95(15):1358.

15. van der Geest IC, de Valk MH, de Rooy JW, Pruszczynski M, Veth RP, Schreuder HW. Oncological and functional results of cryosurgical therapy of enchondromas and chondrosarcomas grade 1 . J Surg Oncol. 2008;98(6):421-6.

16. Verdegaal SH, Brouwers HF, van Zwet EW, Hogendoorn PC, Taminiau AH. Low-grade chondrosarcoma of long bones treated with intralesional curettage followed by application of phenol, ethanol, and bone-grafting. J Bone Joint Surg Am. 2012;94(13):12017.

17. Deckers C, Schreuder BH, Hannink G, de Rooy JW, van der Geest IC. Radiologic follow-up of untreated enchondroma and atypical cartilaginous tumors in the long bones. J Surg Oncol. 2016;114(8): 987-91.

18. Rosenthal DI, Hornicek FJ, Torriani M, Gebhardt MC, Mankin HJ. Osteoid osteoma: percutaneous treatment with radiofrequency energy. Radiology. 2003;229(1):171-5.

19. Weis S, Franke A, Mössner J, Jakobsen JC, Schoppmeyer K. Radiofrequency (thermal) ablation versus no intervention or other interventions for hepatocellular carcinoma. Cochrane Database Syst Rev. 2013;12:CD003046.

20. Katsanos K, Mailli L, Krokidis M, McGrath A, Sabharwal T, Adam A. Systematic review and meta-analysis of thermal ablation versus 
surgical nephrectomy for small renal tumours. Cardiovasc Intervent Radiol. 2014;37(2):427-37.

21. Goetz MP, Callstrom MR, Charboneau JW, et al. Percutaneous image-guided radiofrequency ablation of painful metastases involving bone: a multicenter study. J Clin Oncol. 2004;22(2):300-6.

22. Dierselhuis EF, van den Eerden PJ, Hoekstra HJ, Bulstra SK, Suurmeijer AJ, Jutte PC. Radiofrequency ablation in the treatment of cartilaginous lesions in the long bones: results of a pilot study. Bone Joint J. 2014;96-B(11):1540-5.
23. Hong K, Georgiades C. Radiofrequency ablation: mechanism of action and devices. J Vasc Interv Radiol. 2010;21(8 Suppl):S179-86.

24. Verdegaal SH, van Rijswijk CS, Brouwers HF, et al. MRI appearances of atypical cartilaginous tumour/grade I chondrosarcoma after treatment by curettage, phenolisation and allografting: recommendations for follow-up. Bone Joint J. 2016;98-B(12):1674-81.

25. Hinshaw JL, Lubner MG, Ziemlewicz TJ, Lee FT Jr, Brace CL. Percutaneous tumor ablation tools: microwave, radiofrequency, or cryoablation - what should you use and why? Radiographics. 2014;34(5):1344-62. 